\title{
Mode-matching without root-finding: application to a dissipative silencer*
}

\author{
Jane B. Lawrie ${ }^{\dagger}$
}

School of Information Systems, Computing and Mathematics

Mathematical Sciences, Brunel University, Uxbridge UB8 3PH, UK

jane.lawrie@brunel.ac.uk

Ray Kirby

School of Engineering and Design

Mechanical Engineering, Brunel University, Uxbridge UB8 3PH, UK

ray.kirby@brunel.ac.uk

$21^{\text {st }}$ February, 2006

\begin{abstract}
This article presents an analytic mode-matching approach suitable for modelling the propagation of sound in a two-dimensional, three-part, ducting system. The approach avoids the need to the find roots of the characteristic equation for the middle section of the duct (the component) and is readily applicable to a broad class of problems. It is demonstrated that the system of equations, derived via analytic mode-matching, exhibits certain features which ensure that they can be re-cast into a form that is independent of the roots of the characteristic equation for the component. The precise details of the component are irrelevant to the procedure; it is required only that there exists an orthogonality relation, or similar, for the eigenmodes corresponding to the propagating wave-forms in this region. The method is applied here to a simple problem involving acoustic transmission through a dissipative silencer of the type commonly found in heating ventilation and air-conditioning (HVAC) ducts. With reference to this example, the silencer transmission loss is computed, and the power balance for the silencer is investigated and is shown to be an identity that is necessarily satisfied by the system of equations, regardless of the level of truncation.
\end{abstract}

*Portions of this work were presented in "On analysing a dissipative silencer: a mode-matching approach" Proceedings of IUTAM 2002/04, Liverpool, U.K. July 2002

${ }^{\dagger}$ Corresponding author. 


\section{Introduction}

Guided waves can be observed in a wide range of physical situations. Examples include water waves propagating along a channel, seismological waves travelling through a layer of rock, the transmission of electromagnetic waves along an optical fibre and acoustical waves manifesting as noise in heating ventilation and air conditioning (HVAC) ducts. Very often the physical problems of interest can be formulated as boundary value problems that are amenable to analytic solution methods such as the Wiener-Hopf technique or modematching. A precursor to these methods, however, is accurate knowledge of sufficient admissible wavenumbers. These are usually defined in terms of a characteristic equation, the complexity of which depends on both the equations governing the waveguide media and on the boundary conditions. Only in the simplest of problems can the wavenumbers be expressed in closed form and for problems involving elastic or porous media and/or flexible walled ducts numerical "root-finding" is a normal procedure. Root-finding, whilst tedious, is by no means an impossible task but the difficulties should not be underestimated. Of course, the Wiener-Hopf and mode-matching techniques are not necessarily the only viable analytic solution methods. A wide range of other techniques are available including those based on Green's theorem and a variety of Fourier integral approaches. Some of these approaches successfully avoid the process of root-finding, see for example Huang; ${ }^{4,5}$ however, such methods are not always easily generalised.

In the following discussion attention is restricted to two-dimensional waveguides. Associated with every mode is an axial and at least one transverse wavenumber which are related through the governing equation(s). Further, it is assumed that the waveguide boundary conditions contain only even derivatives in the axial direction. ${ }^{10}$ Then, broadly speaking, the characteristic equation, which will be meromorphic and expressed in terms of the transverse wavenumber, can be broken down into four categories. First, there is the simple trigonometric form, such as that for a rigid walled acoustic duct, for which the roots can be written down in closed form. Second, there are those that involve a linear combination of trigonometric terms and possibly algebraic terms but which have only real parameters and involve only even powers of at most one transverse wavenumber. For such characteristic functions the roots always lie on either the real axis or the imaginary axis. Furthermore, the asymptotic form of the roots is usually known. The characteristic equations for flexible-walled acoustic ducts ${ }^{10}$ are typical of this class. The third class of 
characteristic function is much the same as the second other than it will involve even powers of two related transverse wavenumbers. Waveguides comprising slabs of an elastic material typically have this type of characteristic function. In this case the roots may be real, imaginary or complex yet much may be known about their location ${ }^{1}$ and asymptotic form. The fourth class of characteristic equation has all the properties of those in class three but, in addition, the physical parameters are complex. This class of characteristic equation arises in the study of dissipative silencers in which the absorbent material is modelled as an equivalent fluid.

The latter class of characteristic equation is known to be problematic when it comes to root-finding. This is because the roots do not necessarily lie on or close to any specified curve in the complex plane which makes it difficult to choose appropriate initial guesses for the root-finding algorithm. Further, the complex parameters of the dispersion relation are usually frequency dependent and thus the location of the roots can vary, often significantly and in arbitrary direction, with small variations in frequency. If one desires to find the roots at one specific frequency then usually, with perseverance, this can be done. Then, if one wishes to plot a given physical property against frequency it is usual to adopt a tracking approach. That is, the roots successfully located for frequency $f_{0}$ are used as initial guesses for frequency $f_{0}+\epsilon, \epsilon<<1$. Although this approach increases the likelihood of finding all required roots as frequency varies, it can fail even for frequency shifts of $\epsilon<0.01 \mathrm{~Hz}$. Not only is it difficult to locate all the roots in a specified region of the complex plane at all desired frequencies, but there are very few reliable techniques by which to determine whether all the roots have been found. The Argument Principle is the only rigorous test, but if one or more roots are found to be missing it is of limited help in locating them. Traditional algorithms used for solving this class of characteristic equation include the Newton-Raphson method and variations such as the secant ${ }^{13}$ and Muller's ${ }^{2}$ methods. None of these are robust in the sense that modes are easily missed if the initial guesses are not sufficiently close to the actual root. Although the effect of a missing root on the results depends on the location of the root, it is always undesirable and often the cause of significant inaccuracies. ${ }^{7}$

Analytic solution methods that avoid root-finding are highly desirable but few and far between. Huang, ${ }^{4,5}$ for example, considers a class of problem whereby a membrane is inserted into a finite section, say $-\ell \leq x \leq \ell$, of an otherwise infinite rigid duct. He 
employs a technique whereby the sound field within the duct is represented by an infinite sum of Fourier integrals, each one forced by a velocity distribution $\sin [n \pi(x+\ell) /(2 \ell)]$ on the duct surface for $-\ell \leq x \leq \ell$. The Fourier coefficients of the pressure field are then determined by substituting an evaluated form of the Fourier integrals into the membrane condition. This works well for the class of problem that Huang considers but the method is limited to situations in which the membrane forms part of the duct surface. The method is not applicable to situations whereby there is a structure, such as a finite length membrane, positioned parallel to the duct wall within the fluid region or to situations in which part of the fluid region is layered comprising, for example, alternate layers of fluid and porous material. Further, the extension of Huang's approach to the the situation in which the membrane is replaced by an elastic plate is straightforward only for the case in which the plate edges are pin-jointed. There is a clear need for analytic techniques that can tackle a wide range of such geometries and do not depend on the roots of the characteristic equation.

This article offers an approach by which root-finding can be avoided for a broad class of problem: the propagation of sound in a two-dimensional, three-part ducting system see, for example, figure 1. The class of problem is described in section II and a general mode-matching solution is presented. This solution exhibits certain features that ensure it can always be recast, using a contour integral technique, into a form that is independent of the roots of the characteristic equation for the middle region. This approach completely by-passes the need to solve the characteristic equation, and provides robust and accurate expressions by which to compute any physical quantity of interest. In section III attention is focussed on a particular example: the propagation of sound through a simple silencer comprising a finite length of lined duct. The contour integral technique is demonstrated and a detailed analysis of the power balance is presented. Further analysis proves that the power balance is an identity which, regardless of the severity of the truncation, is automatically satisfied by the system of equations relating the reflection and transmission coefficients. The latter result is well established for non-dissipative systems ${ }^{9,14}$ but, to the authors' knowledge, has not previously been proven for a dissipative system. Numerical results, in terms of transmission loss and power absorbed across each surface of the liner, are presented for two different silencer configurations in section IV. In section $\mathrm{V}$ a discussion of the potential extensions and limitations of this "root-free" approach is 
presented.

\section{The general problem}

In this section the general problem of determining the sound field in a two-dimensional, three-part ducting system is discussed. It is demonstrated that, for the class of problem to be considered, the system of equations derived via mode-matching have a particular structure that ensures that they can always be re-cast into a form that is independent of the roots of the characteristic equation. The system comprises inlet and outlet ducts lying in the regions $0 \leq \bar{y} \leq \bar{b}, \bar{x}<0$ and $0 \leq \bar{y} \leq \bar{b}, \bar{x}>2 \bar{\ell}$ of a Cartesian frame of reference. A "silencer-type" component is sandwiched between them occupying the region $0 \leq \bar{y} \leq \bar{h}$, $0 \leq \bar{x} \leq 2 \bar{\ell}$ where $\bar{h}$ may be greater than, less than or equal to $\bar{b}$. The vertical line segments joining the points $y=h$ to $y=b$ at $x=0$ and $x=2 \ell$ (when $h \neq b$ ) are assumed to be rigid. Thus, the duct geometry is symmetrical about the vertical line $x=\ell$. Figure 1 shows a specific example of a typical three-part ducting system in which $h=b$. The analysis that follows is, however, in no way restricted to this case. A compressible fluid of sound speed $c_{f}$ and density $\rho$ fills the interior of the duct and region exterior to the duct is in vacuo. The incident sound field is assumed to have harmonic time dependence, $e^{+i \omega \bar{t}}$, where $\omega$ is related to the frequency by $\omega=2 \pi f$, and propagates through the fluid in the positive $\bar{x}$ direction towards $\bar{x}=0$.

It is convenient to non-dimensionalise the boundary value problem using typical length and time scales $k^{-1}$ and $\omega^{-1}$ where $\omega=c_{f} k$. Thus, $x=k \bar{x}, y=k \bar{y}$ etc. where the "barred" quantities are dimensional. The time-independent fluid velocity potentials for each duct region are $\phi_{1}(x, y), x<0 ; \phi_{2}(x, y), 0<x<2 \ell$ and $\phi_{3}(x, y), x>2 \ell$ (see figure 1 ). For the inlet and outlet ducts $(x<0$ and $x>2 \ell$ respectively) the velocity potentials are governed by Helmholtz's equation and must satisfy specified boundary conditions at $y=0$ and $y=b$. Thus,

$$
\begin{aligned}
\phi_{1} & =\sum_{j=0}^{\infty} F_{j} Z_{j}(y) e^{-\eta_{j} x}+\sum_{j=0}^{\infty} A_{j} Z_{j}(y) e^{\eta_{j} x} \\
\phi_{3} & =\sum_{j=0}^{\infty} D_{j} Z_{j}(y) e^{-\eta_{j}(x-2 \ell)}
\end{aligned}
$$

where $A_{j}, D_{j}, j=1,2,3 \ldots$ are the complex amplitudes of the reflected and transmitted modes and $\eta_{j}$ is the axial wavenumber of the duct modes. The incident sound field in 
(1) accommodates multi-modal forcing for which the modal amplitudes $F_{n}$ will inevitably depend on the characteristics of the noise source (typically a fan). The eigen-modes $Z_{j}(y)$, $j=0,1,2, \ldots$ depend on the inlet/outlet duct walls which, for example, may be rigid, soft or wave-bearing. In the later case the eigenmodes $Z_{j}(y), j=0,1,2, \ldots$ will satisfy a non-Sturm-Liouville eigensystem and the orthogonality relation (OR) will be of the form (4) together with (5) discussed below. This point is revisited in the section V.

It is assumed that the component lying in the region $0 \leq x \leq 2 \ell$ is of very much more complicated structure than the inlet/outlet ducts, possibly comprising wave-bearing walls and layers of absorbent material. The precise details of the component are irrelevant to the mode-matching procedure, it is required only that there exists an orthogonality relation, or similar, for the eigenmodes corresponding to the the propagating wave-forms in the component region. Under such circumstances the velocity potential in the component region is given by

$$
\phi_{2}=\sum_{j=0}^{\infty}\left(B_{j} e^{-s_{j} x}+C_{j} e^{s_{j} x}\right) Y_{j}(y)
$$

where $B_{j}, C_{j}$ are the complex amplitudes of the waves in the component region. The quantities $s_{j}$ and $Y_{j}(y), j=1,2,3 \ldots$ are the wavenumber of the $j^{\text {th }}$ mode and the corresponding eigenfunction. Although details of the eigensystem are not required for the process described here, it should be noted that the wavenumbers, $s_{j}, j=0,1,2, \ldots$ are defined as the roots of the characteristic equation $K(s)=0$ and that the eigenfunctions must satisfy an OR, or similar, of the form

$$
\left(Y_{n}, Y_{m}\right)=\delta_{m n} E_{n}
$$

where $\delta_{m n}$ is the usual Kronecker delta function. It is worthwhile pointing out that such relationships exist not only for Sturm-Liouville eigensystems but also for systems in which the boundary conditions contain high-order, even derivatives with respect to the axial direction of the waveguide, that is, $x$. (A consequence of the latter point is that $K(-s)=K(s)$.) Such boundary conditions arise if wave-bearing surfaces, such as a membrane or elastic plate, form part of the component. In such cases the OR will comprise the usual integral term together with a linear combination of the eigenfunctions and their derivatives. A typical form for the left hand side of the OR is thus

$$
\left(Y_{n}, Y_{m}\right)=\int_{0}^{d} Y_{n}(y) Y_{m}(y) d y+P\left(\gamma_{n}^{2}, \gamma_{m}^{2}\right) Y_{n}^{\prime}\left(y_{0}\right) Y_{m}^{\prime}\left(y_{0}\right)
$$


where $\gamma_{n}=\left(-1-s_{n}^{2}\right)^{1 / 2}$ and $P(u, v)=P(v, u)$ is a polynomial in the variables $u$ and $v$, the coefficients of which depend on the boundary condition describing the wave-bearing surface which is taken to lie along the line segment $y=y_{0}, 0 \leq x \leq 2 \ell$. A general class of such expressions was derived by Lawrie and Abrahams ${ }^{10}$ and these have subsequently been utilised in a variety of situations. ${ }^{3,11,14}$ Expression (4) is written as an inner-product. This formulation is accurate for Sturm-Liouville systems and also those containing membrane boundaries. For systems with higher-order derivatives present in the boundary conditions the left hand side of (4) will not be an inner product in the usual sense. This, however, is immaterial to the following analysis.

Crucial to the process of expressing the mode-matching equations in root-free form is the knowledge that $E_{n}$ is related to the characteristic equation through an expression of the form

$$
E_{n}=\left.\frac{Y_{n}^{\prime}\left(y_{0}\right)}{2 s_{n}} \frac{d}{d s} K(s)\right|_{s=s_{n}}
$$

where the prime indicates differentiation with respect to $y$ and $y_{0}$ is constant, $0 \leq y_{0} \leq h$. The physical significance of the line segment $y=y_{0}, 0 \leq x \leq 2 \ell$ depends on the details of the component but very often represents an interface between fluid and, for example, a porous material or, as indicated above, the line along which a wave-bearing surface lies. The precise form of $(6)$ is subject to minor variations, sometimes $Y_{n}\left(y_{0}\right)$ appears rather than its derivative, however, $E_{n}$ is invariably proportional to the derivative of the characteristic equation and inversely proportional to the wavenumber.

Equations (1)-(3) give the form of the wave-field in each region of the duct. The modal coefficients $A_{j}-D_{j}, j=0,1,2, \ldots$ are, however, as yet undetermined. Mode-matching is usually achieved by applying continuity of pressure and normal velocity at the interfaces between the component and inlet/outlet duct. This process is now demonstrated. For ease of exposition, the inlet and outlet ducts are assumed to be rigid and plane wave forcing is be assumed (although neither simplification is a requirement of the solution method). Thus, the duct modes are given by $Z_{j}(y)=\cos (j \pi y / b)$ and $F_{j}=\delta_{j 0}$.

It is convenient to make use of the symmetry of the duct geometry and consider separately the symmetric and anti-symmetric sub-problems. In both cases only the left hand side of the system need be considered, and the conditions $\phi_{2 x}(\ell, y)=0$ or $\phi_{2}(\ell, y)=$ 0 are applied along the line of symmetry for the symmetric and antisymmetric cases respectively. There are two continuity conditions to be applied at $x=0$ and two ORs by 
which to do this. Which condition is enforced using which OR depends on the geometry. For $h>b$ continuity of normal velocity is enforced using (4) and continuity of pressure is enforced using the OR for the standard duct modes, $\{\cos (n \pi y / b) \mid n=0,1,2, \ldots\}$. If $h<b$ the situation is reversed whilst for $h=b$ there is a choice. It henceforth assumed, without loss of generality, that $h>b$. Thus, for the symmetric problem, continuity of pressure yields the following expression:

$$
A_{n}^{S}=\frac{4}{b \varepsilon_{n}} \sum_{j=0}^{\infty} B_{j}^{S} \cosh \left(s_{j} \ell\right) R_{n j}-\delta_{n 0} .
$$

Here $A_{n}^{S}$ are the reflection coefficients, $B_{j}^{S}$ are the amplitude of the waves inside the component for this sub-problem and

$$
R_{j m}=\int_{0}^{b} \cos \left(\frac{j \pi y}{b}\right) Y_{m}(y) d y
$$

On using (5), it is found that continuity of normal velocity yeilds

$$
B_{m}^{S}=\frac{1}{2 s_{m} E_{m} \sinh \left(s_{m} \ell\right)}\left\{-i R_{0 m}+\sum_{j=0}^{\infty} A_{j}^{S} \eta_{j} R_{j m}-2 Y_{m}^{\prime}\left(y_{0}\right) \sum_{n=0}^{N} a_{n}^{S} \gamma_{m}^{2 n}\right\} .
$$

It is important to note that for a component with a Sturm-Liouville eigensystem $a_{n}^{S}=0$, $n=0,1, \ldots, N$. In contrast, for one involving, for example, an elastic plate these coefficients are non-zero and must be determined by applying appropriate edge conditions such as zero-displacement and gradient at the plate edges. It is straightforward to eliminate $B_{m}^{s}$ between (9) and (7) to obtain:

$$
A_{m}^{S}=\frac{2}{b \varepsilon_{m}} \sum_{n=0}^{\infty} A_{n}^{S} \eta_{n} \Omega_{n m}-\frac{2 i}{b \varepsilon_{m}} \Omega_{0 m}-\frac{4}{b \varepsilon_{m}} \sum_{n=0}^{N} a_{n}^{S} \sum_{j=0}^{\infty} \frac{\operatorname{coth}\left(s_{j} \ell\right)}{s_{j} E_{j}} \gamma_{j}^{2 n} Y_{j}^{\prime}\left(y_{0}\right) R_{m j}-\delta_{m 0}
$$

where

$$
\Omega_{n m}=\sum_{j=0}^{\infty} \frac{\operatorname{coth}\left(s_{j} \ell\right)}{s_{j} E_{j}} R_{m j} R_{m j}
$$

For the anti-symmetric problem a system of equations with identical structure is obtained, the only difference is that $\Omega_{n m}$ is replaced by $\Lambda_{n m}$ which is given by (11) but with $\operatorname{coth}\left(s_{j} \ell\right)$ replaced by $\tanh \left(s_{j} \ell\right)$. This replacement also applies to any sums on the right hand side of (10). Note that the form of $\Omega_{n m}$ may vary slightly. For example, if continuity of normal velocity and pressure are enforced using the opposite choice of OR to that taken here the wavenumber $s_{j}$ will occur in the numerator of the summand rather than the denominator and this does occur in the example implemented in the next section. The solution to the 
full problem is obtained from the symmetric and anti-symmetric sub-problems simply by noting that $A_{n}=A_{n}^{S}+A_{n}^{A}$ and $D_{n}=A_{n}^{S}-A_{n}^{A}$.

Equation (10) and its anti-symmetric counterpart are of a form that is generic to any three-part ducting system of the class considered here. The systems may be solved by truncation and numerical inversion of the matrix. Accurate evaluation of the quantities $\Omega_{j n}$ and $\Lambda_{j n}$ (and any further sums on the right hand side of (10)) for all required frequencies is crucial to this process but, in their current form, this depends on locating sufficient roots, $s_{j}$. Note, however, that these roots occur only in within these sums and that the quantity $E_{m}$ can be eliminated using (6). Thus, $\Omega_{n m}$ can be expressed as

$$
\Omega_{n m}=2 \sum_{j=0}^{\infty} \frac{\operatorname{coth}\left(s_{j} \ell\right)}{\left.Y_{j}^{\prime}\left(y_{0}\right) \frac{d}{d s} K(s)\right|_{s=s_{j}}} R_{m j} R_{m j} .
$$

Once expressed in this form it can be seen that all such sums exhibit two important features. Firstly, the summands are even functions of $s_{j}$. This is due to the fact that the governing equation (Helmholtz') contains only even powers of both $x$ and $y$, and all boundary conditions for the component region are either Sturm-Liouville or contain only even higher derivatives in $x$. Secondly, each sum has the form of an infinite sum of the residues arising from an odd integrand. It is the latter fact that ensures that each sum can be recast in a form that is independent of the roots, $s_{j}$, of the characteristic equation for the component region. All that is required is to choose an appropriate contour integral. The process is demonstrated in the next section.

\section{A simple dissipative silencer}

In this section the process of expressing $\Omega_{j n}$ and $\Lambda_{j n}$ in root-free form is demonstrated for a two dimensional, three-part ducting system in which the middle component is a simple dissipative silencer of the type that is commonly found in a typical HVAC ducting system. Thus, the section of duct lying in the region $0<\bar{x} \leq 2 \bar{\ell}$ is lined with a porous material which occupies the space $a \leq y \leq b, b>a$. The porous media is modelled as an equivalent fluid with complex speed of propagation $c_{p}$ and complex density $\rho_{p}=Z_{a} / c_{p}$ where $Z_{a}$ is the impedance of the material. Much experimental work has been done on relating $c_{p}$ and $\rho_{p}$ to the bulk acoustic properties of real porous materials and the complex values of these parameters are known in principle. ${ }^{6}$ 


\section{A The mode-matching solution}

The velocity potential in the silencer region $(0 \leq x \leq 2 \ell)$ must satisfy Helmholtz's equation with unit wavenumber in the central passage, $0 \leq y<a$ and with wavenumber $c_{f} / c_{p}$ in the porous media $a<y \leq b$. At the interface between the porous media and the fluid $(y=a)$ it is assumed that the pressure and normal velocity are continuous whilst at the rigid duct walls the normal velocity is zero. The travelling waveforms in a duct of this type are easily determined by using separation of variables. A typical mode travelling in the positive $x$ direction has the form $\phi_{2 n}(x, y)=Y_{n}(y) e^{-s_{n} x}$ where

$$
Y_{n}(y)= \begin{cases}Y_{1 n}(y), & 0 \leq y<a \\ Y_{2 n}(y), & a \leq y \leq b\end{cases}
$$

and the wavenumber $s_{n}$ is defined below as a root of (21). The eigenfunctions $Y_{n}(y)$, $n=0,1,2, \ldots$ are the solutions to the eigensystem:

$$
\begin{aligned}
Y_{1}^{\prime \prime}-\gamma^{2} Y_{1} & =0, \quad 0 \leq y<a ; \\
Y_{1}^{\prime}(0) & =0 ; \\
Y_{2}^{\prime \prime}-\lambda^{2} Y_{2} & =0, \quad a \leq y<b ; \\
Y_{2}^{\prime}(b) & =0 ; \\
Y_{1}(a) & =\beta Y_{2}(a) ; \\
Y_{1}^{\prime}(a) & =Y_{2}^{\prime}(a)
\end{aligned}
$$

where $\beta=\rho_{p} / \rho, \gamma(s)=\left(-1-s^{2}\right)^{1 / 2}$ and $\lambda(s)=\left(\Gamma^{2}-s^{2}\right)^{1 / 2}$ with $\Gamma=i c_{f} / c_{p}$, the branches being chosen such that $\gamma(0)=+i$ and $\lambda(0)=+\Gamma$.

It is readily shown that

$$
Y_{n}(y)=\left\{\begin{array}{ll}
\cosh \left(\gamma_{n} y\right), & 0 \leq y<a \\
\frac{\gamma_{n} \sinh \left(\gamma_{n} a\right)}{\lambda_{n} \sinh \left[\lambda_{n} d\right]} \cosh \left[\lambda_{n}(y-b)\right], & a \leq y \leq b
\end{array} .\right.
$$

Here $d=b-a, \gamma_{n}=\gamma\left(s_{n}\right), \lambda_{n}=\lambda\left(s_{n}\right)$ and $s_{n}, n=0,1,2, \ldots$ are those roots of the dispersion relation $K(s)=0$ with $\Re\left(s_{n}\right) \geq 0$, where

$$
K(s)=\cosh (\gamma a)+\frac{\beta \gamma \sinh (\gamma a)}{\lambda \sinh [\lambda(b-a)]} \cosh [\lambda(b-a)] .
$$

Note that the roots $s_{n}, n=0,1,2, \ldots$ are numbered by increasing real part. Thus, the mode with wavenumber $s_{0}$ is the least attenuated. 
The orthogonality relation ${ }^{8}$ for this set of eigenfunctions is

$$
\int_{0}^{a} Y_{1 n} Y_{1 m} d y+\beta \int_{a}^{b} Y_{2 n} Y_{2 m} d y=\delta_{m n} E_{n}
$$

where $\delta_{m n}$ is the usual Kronecker delta and $E_{n}$ is given by (6) with $y_{0}=a$.

The velocity potentials $\phi_{j}, j=1,2,3$ are given by (1)-(2) and (3) with $Z_{n}(y)=$ $\cos (n \pi y / b), n=0,1,2, \ldots$ and $\eta_{j}=\left(j^{2} \pi^{2} / b^{2}-1\right)^{1 / 2}$ with $\eta_{0}=i$, these definitions being appropriate for the choice of time dependence. Numerical results for both plane wave forcing and a multi-modal incident field will be presented in the next section. Thus, the following analysis is carried out under the assumption of multi-modal forcing, but without stating explicitly the form of the modal amplitudes. The appropriate form for both types of forcing are stated in section IV. The mode-matching procedure described in section II yields the following systems of equations:

$$
\chi_{n}=F_{n} \eta_{n}^{1 / 2}-\frac{2}{b \varepsilon_{n} \eta_{n}^{1 / 2}} \sum_{j=0}^{\infty}\left(\chi_{j}+F_{j} \eta_{j}^{1 / 2}\right) \frac{\Lambda_{j n}}{\eta_{j}^{1 / 2}}
$$

and

$$
\psi_{n}=F_{n} \eta_{n}^{1 / 2}-\frac{2}{b \varepsilon_{n} \eta_{n}^{1 / 2}} \sum_{j=0}^{\infty}\left(\psi_{j}+F_{j} \eta_{j}^{1 / 2}\right) \frac{\Omega_{j n}}{\eta_{j}^{1 / 2}}
$$

where $\chi_{n}=\left(A_{n}+D_{n}\right) \eta_{n}^{1 / 2} ; \psi_{n}=\left(A_{n}-D_{n}\right) \eta_{n}^{1 / 2}$ and $R_{j m}$ is given by (8). Note that, the explicit form for $R_{j m}$ for this problem is given in equation (33). For this coupled system of equations

$$
\Lambda_{j n}=\sum_{m=0}^{\infty} \frac{s_{m}}{E_{m}} \tanh \left(s_{m} \ell\right) R_{j m} R_{n m}
$$

and $\Omega_{j n}$ is given by (25) with $\tanh \left(s_{m} \ell\right)$ replaced by $\operatorname{coth}\left(s_{m} \ell\right)$. The coefficients $B_{n}$ and $C_{n}, n=0,1,2, \ldots$ are expressed in terms of $A_{n}$ and $D_{n}$ by

$$
B_{m}+C_{m}=\frac{1}{E_{m}} \sum_{j=0}^{\infty}\left(A_{j}+F_{j}\right) R_{j m}
$$

and

$$
B_{m} e^{-2 s_{m} \ell}+C_{m} e^{2 s_{m} \ell}=\frac{1}{E_{m}} \sum_{j=0}^{\infty} D_{j} R_{m j} .
$$

\section{B Recasting the matrix elements}

As discussed in section II, expression (25) can be recast in a form whereby the summation takes place over eigenvalues that can be expressed in closed form. The key is expression 
(6) which relates the quantity $E_{n}$ to the derivative of the dispersion relation. This enables (25) to be recognised as the sum over a family of poles for a carefully selected integral. The appropriate integral is

$$
I_{j m}=\lim _{X \rightarrow \infty} \int_{-i X}^{i X} \frac{\ell \Upsilon(s) L_{j}(s) L_{m}(s)}{\operatorname{coth}(s \ell)} d s
$$

where $X>>1$ is real. The quantities $\Upsilon(s)$ and $L_{j}(s)$ are defined by

$$
\Upsilon(s)=\frac{s^{2} \gamma \sinh (\gamma a)}{\ell K(s)}
$$

and

$$
L_{j}(s)=\frac{P_{j}(s)}{\gamma^{2}+(j \pi / b)^{2}}-\frac{Q_{j}(s)}{\lambda^{2}+(j \pi / b)^{2}},
$$

with $\gamma=\left(-1-s^{2}\right)^{1 / 2}$ and $\lambda=\left(\Gamma^{2}-s^{2}\right)^{1 / 2}$, and

$$
\begin{aligned}
P_{j}(s) & =\cos \left(\frac{j \pi a}{b}\right)+\frac{j \pi}{b} \sin \left(\frac{j \pi a}{b}\right) \frac{\cosh (\gamma a)}{\gamma \sinh (\gamma a)} \\
Q_{j}(s) & =(-1)^{j}\left\{\cos \left(\frac{j \pi d}{b}\right)+\frac{j \pi}{b} \sin \left(\frac{j \pi d}{b}\right) \frac{\cosh (\lambda d)}{\lambda \sinh (\lambda d)}\right\} .
\end{aligned}
$$

The choices of $P_{j}(s)$ and $Q_{j}(s)$ are such that $L_{j}(s)$ does not have poles when $\gamma^{2}+(j \pi / b)^{2}=$ 0 or $\lambda^{2}+(j \pi / b)^{2}=0$ (provided $\left.j>0\right)$. Further, although $Q_{j}(s)$ seems to be written in a slightly cumbersome form, it is this form that ensures that $Q\left(\tau_{j}\right)=0$ by inspection (i.e. without recourse to any trigonometric relations) where $\tau_{j}=\left(\Gamma^{2}+j^{2} \pi^{2} / b^{2}\right)^{1 / 2}$. Note that, the function $L_{j}(s)$ is related to the quantity $R_{j n}$, see (8), by

$$
\gamma_{n} \sinh \left(\gamma_{n} a\right) L_{j}\left(s_{n}\right)=R_{j n}
$$

The path of integration in (28) lies along the imaginary axis and is indented to the left(right) of any poles on the upper(lower) half of the imaginary axis. Therefore, since the integrand is an odd function of $s, I_{j m}=0$ as $|X| \rightarrow \infty$. Note also that the integrand is $0\left(s^{-3}\right)$ as $|s| \rightarrow \infty$. Thus, on deforming the path of integration onto a semi-circular $\operatorname{arc}$ of radius $X$ in the right-hand half plane, the sum of the residues of all the poles crossed is zero as $X \rightarrow \infty$. The integrand has families of poles when: (i) $K(s)=0$, i.e. $s=s_{n}$; (ii) $\cosh (s \ell)=0$, i.e. $s=\sigma_{1 n}=(2 n+1) i \pi /(2 \ell) ; \quad($ iii $) \sinh (\lambda d)=0$, i.e. $s=\nu_{n}=\left(\Gamma^{2}+n^{2} \pi^{2} / d^{2}\right)^{1 / 2} ;$ (iv) $\gamma \sinh (\gamma a)=0$, i.e. $s=\theta_{n}=\left(n^{2} \pi^{2} / a-1\right)^{1 / 2}$ where $d=b-a$ and, in each case, $n=0,1,2, \ldots$. Note that, as mentioned above, the forms of $P_{j}(s)$ and $Q_{j}(s)$ ensure that the quantity $L_{j}(s)$ has no poles when $\gamma^{2}+(j \pi / b)^{2}=0$ 
or $\lambda^{2}+(j \pi / b)^{2}=0$ provided $j>0$. The cases $j=0, m \neq 0 ; m=0, j \neq 0$ and $m=j=0$, however, need separate consideration. For these values of $m$ and $j$ the families of poles denoted (ii) and (iii) above vanish leaving, in both cases, only the simple pole corresponding to $n=0$.

The first family of poles yields $\Lambda_{j m}$ and, on evaluating all the other pole contributions, it is found that

$$
\begin{aligned}
\frac{1}{2} \Lambda_{j m} & =-\sum_{n=0}^{\infty} \Upsilon\left(\sigma_{1 n}\right) L_{j}\left(\sigma_{1 n}\right) L_{m}\left(\sigma_{1 n}\right) \\
& +\frac{(-1)^{j+m}}{\beta d} \sum_{n=0}^{\infty} \frac{\nu_{n} V_{j n}(d) V_{m n}(d)}{\varepsilon_{n} \operatorname{coth}\left(\nu_{n} \ell\right)}+\sum_{n=0}^{\infty} \frac{\theta_{n} V_{j n}(a) V_{m n}(a)}{a \varepsilon_{n} \operatorname{coth}\left(\theta_{n} \ell\right)}
\end{aligned}
$$

where

$$
V_{m n}(x)= \begin{cases}\frac{\frac{m \pi}{b} \sin \left(\frac{m \pi x}{b}\right)}{\left[(m \pi / b)^{2}-(n \pi / x)^{2}\right]}, & m \neq n, m x \neq n b \\ x, & m=n=0 \\ \frac{x}{2}(-1)^{m+n}, & m x=n b, m \neq 0\end{cases}
$$

with $x$ real. Note first that the second sum on the right hand side of (34) is of identical structure to the third sum but with $\theta_{n}$ replaced by $\nu_{n}$ and $a$ by $d=b-a$. $\operatorname{Had} Q_{j}(s) \operatorname{not}$ been defined as in (32) the trigonometric terms in the numerator of the second summand would have contained $a$ rather than $d$ producing an incorrect value when $m d=n b$ and $m=j=0$. Note secondly that, for the cases $j=0, m \neq 0 ; m=0, j \neq 0$ and $m=j=0$ the second and third sums of (34) both reduce to one term. This is consistent with the vanishing of the families of poles denoted by (ii) and (iii) for these values of $m$ and $j$ as discussed above.

A similar expression for $\Omega_{j m}$ can be obtained using the integral (28) but with the term $\operatorname{coth}(s \ell)$ in the denominator of the integrand replaced with $\tanh (s \ell)$. The expression for $\Omega_{j m}$ is of identical structure to (34) except that every occurence of coth is replaced by tanh and every occurence of $\sigma_{1 n}$ is replaced by $\sigma_{2 n}=n \pi i / \ell, n=0,1,2, \ldots$ Having recast the matrix elements into a form that is independent of the roots of (21), equations (23) and (24) many be truncated and solved numerically to determine the amplitudes $A_{n}$ and $D_{n}$ 


\section{The Power Balance}

In the previous section it was shown that it is possible to fully determine the complex amplitudes $A_{n}$ and $D_{n}$ without solving the complicated dispersion relation $K(s)=0$ where $K(s)$ is given by (21). Clearly the incident, reflected and transmitted powers are independent of the roots of the dispersion relation and can thus easily be determined. The expressions for these quantities are well known, that for the incident power is

$$
P_{\text {Inc }}=\frac{1}{2} \Re\left\{\sum_{m=0}^{\infty}\left|F_{m}\right|^{2} i \eta_{m}^{*} \varepsilon_{m}\right\},
$$

where* indicates the complex conjugate. The expressions for the reflected and transmitted powers, $P_{\text {ref }}$ and $P_{\text {Trans }}$, are identical but with $F_{m}, m=0,1,2, \ldots$ replaced by $A_{m}$ and $D_{m}$ respectively. Note that these expressions are given in the form of power flux per unit height. Further, $F_{m}, m=0,1,2, \ldots$ are defined such that the incident power as given by (36) is unity.

It is a trivial matter to deduce that the power absorbed by the layer of lining is $P_{\text {Inc }}-P_{\text {Ref }}-P_{\text {Trans }}$. Such an expression, however, is inadequate in two respects. First, it does not give any indication of the power flux across each of the three surfaces of the absorbent lining. Second, it does not complete the power balance and thus, the opportunity to implement an algorithmic check on the algebra is missed. In fact, it is straightforward to calculate the power flux across the vertical surfaces at $a \leq y \leq b$, $x=0$ and $a \leq y \leq b, x=2 \ell$ using the non-dimensional flux integral

$$
P=\frac{1}{b} \Re\left\{-i \int_{a}^{b} \phi_{j}\left(\frac{\partial \phi_{j}}{\partial x}\right)^{*} d y\right\}
$$

where $j=1$ for the surface at $x=0$ and $j=3$ for the surface at $x=2 \ell$. It is found that the power absorbed across $x=0$ is

$$
P_{x=0}=\Re\left\{\frac{i}{b} \sum_{n=0}^{\infty} \sum_{j=0}^{\infty}\left(F_{n}+A_{n}\right)\left(F_{j}-A_{j}\right)^{*} \eta_{j}^{*} I_{j n}\right\},
$$

whilst that absorbed across the surface at $x=2 \ell$ is

$$
P_{x=2 \ell}=\Re\left\{-\frac{i}{b} \sum_{n=0}^{\infty} \sum_{j=0}^{\infty} D_{n} D_{j}^{*} \eta_{j}^{*} I_{j n}\right\}
$$

where

$$
I_{j n}=\int_{a}^{b} \cos \left(\frac{j \pi y}{b}\right) \cos \left(\frac{n \pi y}{b}\right) d y
$$


which is easily evaluated and independent of the roots $s_{n}, n=0,1,2, \ldots$ The expressions for $P_{x=0}$ and $P_{x=2 \ell}$ given above were calculated using $\phi_{1}$ and $\phi_{3}$ respectively and are the simplest formulae for the power flux across the vertical surfaces. It would, however, have been equally appropriate to use $\phi_{2}$ (in which case (37) must be multiplied by $\beta=\rho_{p} / \rho$ to account for the difference in density of the absorbent lining and the acoustic medium). This yields equivalent expressions in terms of the amplitudes $B_{n}$ and $C_{n}$. It is found that

$$
P_{x=0}=\Re\left\{i \sum_{n=0}^{\infty} \sum_{m=0}^{\infty} \frac{\Delta_{m n} G_{n m}^{*}(0)}{\lambda_{m}^{2}-\left(\lambda_{n}^{*}\right)^{2}}\right\} ;
$$

where

$$
\Delta_{m n}=\frac{\beta}{\beta^{*}} \gamma_{m} \sinh \left(\gamma_{m} a\right) \cosh \left(\gamma_{n}^{*} a\right)-\gamma_{n}^{*} \sinh \left(\gamma_{n}^{*} a\right) \cosh \left(\gamma_{m} a\right)
$$

and

$$
G_{m n}(x)=s_{m}\left(C_{m} e^{2 s_{m} x}-B_{m} e^{-2 s_{m} x}\right)\left(B_{n}^{*} e^{-2 s_{n}^{*} x}+C_{n}^{*} e^{2 s_{n}^{*} x}\right) .
$$

The power flux across the surface at $x=2 \ell$ is given by $(41)$ with $G_{m n}^{*}(0)$ replaced by $-G_{m n}^{*}(\ell)$. These expressions depend on the wavenumbers $s_{n}$ and the amplitudes $B_{n}$ and $C_{n}$, however, they can be recast in a form that is independent of these quantities. The new forms depend only on $A_{n}, D_{n}, F_{n}$ and the independent eigenvalues $\nu_{n}, \sigma_{1 n}$ and $\sigma_{2 n}$ defined in section III B. Details of the rearrangement are given in the appendix where it is shown that

$$
P_{x=0}=\Re\left\{\frac{i}{b} \sum_{j=0}^{\infty} \sum_{n=0}^{\infty}\left[\tilde{A}_{j}^{*} \tilde{A}_{n} S 1_{j n}-\tilde{A}_{j}^{*} D_{n} S 2_{j n}\right]\right\}
$$

and

$$
P_{x=2 \ell}=\Re\left\{\frac{i}{b} \sum_{j=0}^{\infty} \sum_{n=0}^{\infty}\left[D_{j}^{*} D_{n} S 1_{j n}-\tilde{A}_{n} D_{j}^{*} S 2_{j n}\right]\right\}
$$

where $\tilde{A}_{j}=\left(A_{j}+F_{j}\right)$ and

$$
S 1_{j n}=\sum_{m=0}^{\infty}\left(-\frac{\Upsilon\left(\sigma_{m}\right) L_{n}\left(\sigma_{m}\right) Q_{j}\left(\sigma_{m}\right)}{\lambda^{2}\left(\sigma_{m}\right)+(j \pi / b)^{2}}-\frac{2(-1)^{n+j}}{\beta d} \frac{\nu_{m} V_{n m}(d) V_{j m}(d)}{\varepsilon_{m} \tanh \left(2 \nu_{m} \ell\right)}\right)
$$

and

$$
S 2_{j n}=\sum_{m=0}^{\infty}\left(\frac{\Upsilon\left(\sigma_{m}\right) L_{n}\left(\sigma_{m}\right) Q_{j}\left(\sigma_{m}\right)}{(-1)^{m+1}\left[\lambda^{2}\left(\sigma_{m}\right)+(j \pi / b)^{2}\right]}-\frac{2(-1)^{n+j}}{\beta d} \frac{\nu_{m} V_{n m}(d) V_{j m}(d)}{\varepsilon_{m} \sinh \left(2 \nu_{m} \ell\right)}\right) .
$$

Note that, $\sigma_{m}=i m \pi /(2 \ell)$ which comprises both $\sigma_{1 m}$ and $\sigma_{2 m}$ as defined in section III B.

The power flux across the horizontal surface of the lining can be expressed only in terms of $\phi_{2}$. The appropriate, non-dimensional, flux integral can be evaluated to obtain

$$
P_{y=a}=\Re\left\{\frac{i}{b} \sum_{m=0}^{\infty} \sum_{n=0}^{\infty} \frac{\cosh \left(\gamma_{m} a\right) \gamma_{n}^{*} \sinh \left(\gamma_{n}^{*} a\right)}{s_{m}^{2}-\left(s_{n}^{*}\right)^{2}}\left[G_{n m}^{*}(\ell)+G_{m n}(0)-G_{m n}(\ell)-G_{n m}^{*}(0)\right]\right\} .
$$


This expression can also be re-expressed in a form that is independent of $B_{m}, C_{m}$ and $s_{m}$. The new form depends on $A_{n}, D_{n}, F_{n}$ and the independent eigenvalues $\theta_{n}, \sigma_{1 n}$ and $\sigma_{2 n}$ defined in section III B. Again, details of the rearrangement, which involves some complicated manipulations, are presented in the appendix. The appropriate form for the power flux across the horizontal surface of the lining is stated here as

$$
P_{y=a}=\Re\left\{\frac{i}{b}\left(\sum_{n=0}^{\infty} \sum_{j=0}^{\infty}\left[\tilde{A}_{n} D_{j}^{*}+D_{n} \tilde{A}_{j}^{*}\right] S 3_{j n}-\left[D_{n} D_{j}^{*}+\tilde{A}_{n} \tilde{A}_{j}^{*}\right] S 4_{j n}\right)\right\}
$$

where

$$
S 3_{j n}=\sum_{m=0}^{\infty}\left(\frac{\Upsilon\left(\sigma_{m}\right) L_{n}\left(\sigma_{m}\right) P_{j}\left(\sigma_{m}\right)}{(-1)^{m+1}\left[\gamma^{2}\left(\sigma_{m}\right)+(j \pi / b)^{2}\right]}+\frac{2 \theta_{m} V_{n m}(a) V_{j m}(a)}{a \varepsilon_{m} \sinh \left(2 \theta_{m} \ell\right)}\right)
$$

and

$$
S 4_{j n}=\sum_{m=0}^{\infty}\left(-\frac{\Upsilon\left(\sigma_{m}\right) L_{n}\left(\sigma_{m}\right) P_{j}\left(\sigma_{m}\right)}{\gamma^{2}\left(\sigma_{m}\right)+(j \pi / b)^{2}}+\frac{2 \theta_{m} V_{n m}(a) V_{j m}(a)}{a \varepsilon_{m} \tanh \left(2 \theta_{m} \ell\right)}\right)
$$

with $\sigma_{m}=i m \pi /(2 \ell)$.

It is not difficult to show that $S 4_{j n}-S 1_{j n}=\left\{\Lambda_{j n}+\Omega_{j n}\right\} / 2$ and $S 3_{j n}-S 2_{j n}=$ $\left\{\Omega_{j n}-\Lambda_{j n}\right\} / 2$. It follows from (44), (45) and (49) that the total power absorbed by the silencer lining is given by

$$
P_{A b s}=\Re\left\{\frac{-i}{2 b}\left(\sum_{j=0}^{\infty} \sum_{n=0}^{\infty}\left[\tilde{A}_{j}^{*} \tilde{A}_{n}+D_{j}^{*} D_{n}\right]\left(\Lambda_{j n}+\Omega_{j n}\right)+\left[D_{j}^{*} \tilde{A}_{n}+\tilde{A}_{j}^{*} D_{n}\right]\left(\Lambda_{j n}-\Omega_{j n}\right)\right)\right\} .
$$

This expression has been calculated directly from the flux integrals and is independent of the reflected and transmitted powers. Thus, the power balance can now be stated as

$$
P_{\text {Ref }}+P_{\text {Trans }}+P_{\text {Abs }}=P_{\text {Inc }}
$$

where $P_{\text {Inc }}, P_{\text {Ref }}, P_{\text {Trans }}$ and $P_{\text {Abs }}$ are given by (36) and (52) respectively.

For problems involving acoustic transmission in non-dissipative conditions, it is well established that the systems of equations derived via mode-matching or similar methods automatically satisfy the power balance regardless of the level of truncation used during numerical solution. ${ }^{9,14}$ Further, Warren et. al. ${ }^{14}$ demonstrate that even if the system of equations is truncated radically, so that fewer equations are retained than the number of cut-on modes, the power balance is still satisfied! (Although clearly, in this case, the distribution of power between the reflected and transmitted components will be incorrect.) This indicates that, whilst it is necessary that the various components of power should 
balance this, in itself, is not sufficient to ensure that the numerical solution obtained via truncation and inversion represents the actual solution to the physical problem under consideration. The authors are not aware of any equivalent results in the literature for a dissipative system and, in view of the importance of this issue, it is now shown that the system of equations, (23) - (25), automatically satisfies the power balance regardless of level of truncation. It is assumed that the system of equations is truncated to $T+1$ terms where $T>0$. Thus, on adding and subtracting (23) and (24), it is found that

$$
A_{m} \eta_{m}=F_{m} \eta_{m}-\frac{1}{b \varepsilon_{m}} \sum_{j=0}^{T}\left[\tilde{A}_{j}\left(\Lambda_{j m}+\Omega_{j m}\right)+D_{j}\left(\Lambda_{j m}-\Omega_{j m}\right)\right]
$$

and

$$
D_{m} \eta_{m}=-\frac{1}{b \varepsilon_{m}} \sum_{j=0}^{T}\left[\tilde{A}_{j}\left(\Lambda_{j m}-\Omega_{j m}\right)+D_{j}\left(\Lambda_{j m}+\Omega_{j m}\right)\right]
$$

where $m=0,1,2, \ldots, T$ and $\tilde{A}_{j}=\left(A_{j}+F_{j}\right)$. Now the sum of the reflected and transmitted powers can be written as

$$
P_{\text {Ref }}+P_{\text {Trans }}=\frac{1}{2}\left\{-i \sum_{m=0}^{T}\left[A_{m}^{*}\left(A_{m} \eta_{m}\right) \varepsilon_{m}+D_{m}^{*}\left(D_{m} \eta_{m}\right) \varepsilon_{m}\right]\right\}
$$

and on using (54) and (55), this becomes

$$
\begin{aligned}
P_{\text {Ref }}+P_{\text {Trans }} & =\frac{1}{2} \Re\left\{-i\left(\sum_{m=0}^{T} A_{m}^{*} F_{m} \eta_{m} \varepsilon_{m}\right.\right. \\
& -\frac{1}{b} \sum_{m=0}^{T} \sum_{j=0}^{T}\left[A_{m}^{*} \tilde{A}_{j}+D_{m}^{*} D_{j}\right]\left(\Lambda_{j m}+\Omega_{j m}\right) \\
& \left.\left.\left.-\frac{1}{b} \sum_{m=0}^{T} \sum_{j=0}^{T}\left[A_{m}^{*} D_{j}+D_{m}^{*} \tilde{A}_{j}\right]\left(\Lambda_{j m}-\Omega_{j m}\right)\right]\right)\right\} .
\end{aligned}
$$

Since only the real part is of interest, the first term on the right hand side of (57) can be replaced with its conjugate. Then, on using (54) to eliminate $A_{m}$ from this term, it is found that

$$
\begin{aligned}
P_{\text {Ref }}+P_{\text {Trans }} & =\frac{1}{2} \Re\left\{i \sum_{m=0}^{T} \eta_{m}^{*} F_{m}^{*} F_{m} \varepsilon_{m}\right. \\
& \left.-\frac{i}{b} \sum_{m=0}^{T} \sum_{j=0}^{T} \frac{\eta_{m}^{*}}{\eta_{m}}\left[F_{m}^{*} \tilde{A}_{j}\left(\Lambda_{j m}+\Omega_{j m}\right)+D_{j} F_{m}^{*}\left(\Lambda_{j m}-\Omega_{j m}\right)\right]\right\} \\
& +\frac{1}{2} \Re\left\{\frac{i}{b} \sum_{m=0}^{T} \sum_{j=0}^{T}\left[A_{m}^{*} \tilde{A}_{j}+D_{m}^{*} D_{j}\right]\left(\Lambda_{j m}+\Omega_{j m}\right)\right. \\
& \left.\left.+\frac{i}{b} \sum_{m=0}^{T} \sum_{j=0}^{T}\left[A_{m}^{*} D_{j}+D_{m}^{*} \tilde{A}_{j}\right]\left(\Lambda_{j m}-\Omega_{j m}\right)\right]\right\}
\end{aligned}
$$


On referring to (59) it is seen that $F_{j}=0$ for $j \geq M_{I}$ where $M_{I}$ is the number of cut-on modes, whilst $\eta_{j}$ is imaginary for $j<M_{I}$. Thus, without loss of generality the quantity $\eta_{m}^{*} / \eta_{m}$ on the right hand side of (58) can be replaced by -1 . Then, on collecting together the terms involving $\Lambda_{j m} \pm \Omega_{j m}$, it becomes apparent that the right hand side of (58) comprises the incident power and that absorbed by the silencer, see (36) and (52), where both expressions are truncated to $T+1$ terms. Hence, it is shown that the power balance is an algebraic identity and in no way guarantees that the numerical solution has converged to that representing the physical problem.

\section{Numerical Results}

All the graphical results presented in this section are calculated using the root free expressions given in section III. Two silencer configurations are studied. These are identical in height $(\bar{b}=0.6 \mathrm{~m})$ and half-length $(\bar{\ell}=1.5 \mathrm{~m})$ and are lined with the same absorbent material. The difference lies in the depth of the absorbent liner. For silencer 1 the absorbent layer is thick at $0.45 \mathrm{~m}$, thus $\bar{a}=0.15 \mathrm{~m}$. For silencer 2 the depth of the absorbent layer is much thinner at $0.15 \mathrm{~m}$ so that $\bar{a}=0.45 \mathrm{~m}$. The absorbent material is characterised by the regression formulae of Delany and Bazley ${ }^{6,7}$ with flow resistivity of $8000 \mathrm{rayl} / \mathrm{m}$. Note, however, that the formulae of Delany and Bazley are known to be invalid at low frequency and so the semi-empirical correction of Kirby and Cummings ${ }^{6}$ are then used.

The usual measure of performance for a dissipative silencer is transmission loss: $\mathcal{L}=$ $-10 \log _{10}\left(P_{\text {Trans }} / P_{\text {Inc }}\right)$ where $P_{\text {Trans }}$ and $P_{\text {Inc }}$ are given by $(36)$. The incident field as defined by (1) may be either plane wave, in which case the modal amplitudes are given by $F_{j}=\delta_{j 0}$, or multi-modal. Mechel ${ }^{12}$ suggests that "equal modal energy density" (EMED) is the most plausible form of multi-modal forcing for this class of system and, under this assumption, the modal amplitudes are given by

$$
F_{j}^{2}= \begin{cases}\frac{2 i}{\varepsilon_{j} \sum_{m=0}^{M_{I}-1} \eta_{m}}, & j<M_{I} \\ 0, & j \geq M_{I}\end{cases}
$$

where $\varepsilon_{j}=2$ for $j=0$ and 1 otherwise. Note that, in (59) $M_{I}$ is the number of waves cut-on in the inlet duct and will depend on $b=k \bar{b}$.

Figure 2 shows transmission loss against frequency for silencer 1. Both EMED and plane wave forcing are shown. At frequencies below $286 \mathrm{~Hz}$ the two curves overlie as is to 
be expected since EMED reduces to plane wave forcing below the first cut-on frequency. Thereafter the transmission loss is slightly higher for plane wave forcing, but the difference is at most 6 decibels. The results presented in Figure 2 are validated in Table I where, for seven specified frequencies, the value of the transmission loss obtained using the root-free method is compared both with that obtained using conventional "rooty" mode-matching (i.e. solving the characteristic equation in order to obtain sufficient wavenumbers, $s_{m}$, by which to accurately evaluate the quantities $\Omega_{j n}$ and $\Lambda_{j n}$ ) and a finite-element based point-collocation method. ${ }^{7}$ All three methods show good agreement.

The power flux across each face of silencer 1, for plane wave and EMED forcing, are shown in figures 3 and 4 respectively. It is interesting to note that, although the overall transmission loss is not vastly different between the two types of forcing, the silencer surface primarily involved in the sound absorption depends on the type of forcing. For plane wave forcing (figure 3) it is the front face of the lining, i.e. the surface lying along $x=0, a \leq y \leq b$, across which the power flux is the greatest, although the flux across horizontal surface, i.e. that lying along $y=a, 0 \leq x \leq 2 \ell$, is also significant. Whereas for EMED forcing (figure 4), it is clear that the power flux across the horizontal face of the silencer is the greatest. In this case, particularly at frequencies greater that $1000 \mathrm{~Hz}$, the energy absorbed across the front face of the silencer is relatively insignificant. For both forcing mechanisms and indeed both silencer configurations, the flux across rear face of the silencer, i.e. that lying along $x=2 \ell, a \leq y \leq b$, is negligible. Indeed as the frequency tends towards zero, energy actually leaks through this surface which manifests as a negative flux. The amount of power reflected for the two forcing mechanisms is similar, although the characteristic spikes at each cut-on frequency are more apparent in figure 4 (EMED forcing).

Figure 5 shows transmission loss against frequency for silencer 2. Again, both EMED and plane wave forcing are shown. As for silencer 1, the two curves overlie for frequencies below $286 \mathrm{~Hz}$ but, in this case, they also remain very close for frequencies up to $850 \mathrm{~Hz}$. Thereafter, the two curves diverge but the maximum difference is still in the region of 5 decibels. There are two points to be noted. First, at all frequencies, the transmission loss for silencer 2 is significantly less than that of silencer 1 . This is to be expected since silencer 2 has the thinner lining of the two silencers. Second, for this silencer the transmission loss is slightly higher for EMED forcing as opposed to plane wave. Again, the 
results presented in Figure 5 are validated, in Table II, by comparison with the "rooty" approach and point-collocation.

Figures 6 and 7 show the power flux across the component surfaces of the silencer for plane wave and EMED forcing respectively. For plane wave forcing both the front and the horizontal faces of the silencer are proactive i.e. the power flux across them is significant. It cannot now be said that the front face is the most proactive. This is to be expected since the silencer lining is comparatively thin and there is, therefore, less sound incident directly onto the front face than with the thicker lining of silencer 1. For EMED forcing, however, it is clear that the horizontal surface accounts for the vast majority of the transmission loss as it does for silencer 1 .

\section{Discussion}

It has been demonstrated that a broad class of problem involving the transmission of sound through a two-dimensional, three-part ducting system can be successfully solved using analytic mode-matching, but without explicit knowledge of any of the roots of the characteristic equation for the "middle" region. Here the method was implemented for a three-part system comprising rigid inlet and outlet ducts with simple silencer sandwiched between them. The systems of equations obtained via mode-matching were recast, using a contour integral technique, into a form that is independent of the roots to the characteristic equation for the silencer region. Robust and accurate root-free expressions by which to compute all the physical quantities of interest were obtained.

In order to use the method described in this article it is required only that there exists an appropriate orthogonality relation for the eigenfunctions of the "middle" or "component" region. For problems in which the inlet/oulet ducts are acoustically hard or soft the systems of equations obtained via mode-matching can be cast into a form that involves no root-finding. For situations in which the inlet/oulet ducts are bounded by wave-bearing surfaces such as a membrane of elastic plate, however, the approach is still of value. The underlying eigensystems for such inlet/oulet ducts are non-Sturm-Liouville but are well studied. ${ }^{3,10}$ Further, although the admissible wavenumbers must be determined numerically, this task is not usually onerous since the roots to the characteristic equation are known to lie only on either the real or imaginary axis and can be located with relatively 
little effort. Thus, for a three-part ducting system comprising inlet/outlet ducts with wavebearing boundaries and a middle component of more complicated structure (possibly comprising both wave-bearing boundaries and layers of porous material) this method will by-pass root-finding for the middle region thereby significantly reducing the overall burden of root-finding. Furthermore, it seems likely that this approach can be extended to three-part ducting systems with circular cylindrical geometry comprising rigid inlet/outlet ducts. All that is required is that a suitable OR exists for the component region.

A minor disadvantage is that this approach, although highly accurate, is computationally slower that the root finding alternative. This disadvantage may be offset, however, against the advantage of eliminating the inaccuracies that can arise due to missing roots. This is of particular importance when plotting physical quantities, such as transmission loss, against frequency.

\section{A Calculation of the absorbed power}

In section III $\mathrm{C}$ the power absorbed across each of the three faces of the silencer lining was given, in terms of the roots $s_{n}, n=0,1,2, \ldots$ of the dispersion relation, by (41). This expression can be recast into forms that depend only on $A_{n}, D_{n}, \sigma_{n}=i n \pi /(2 \ell)$ and $\nu_{n}=\left(\Gamma^{2}+n^{2} \pi^{2} / d^{2}\right)^{1 / 2}$ and which are, therefore, independent of $s_{n}$. Consider first the vertical faces, the first step in recasting (41) is to eliminate $B_{n}$ and $C_{n}$ using (26), (27) and the following expressions

$$
\begin{aligned}
B_{n}-C_{n} & =\frac{\operatorname{coth}\left(2 s_{n} \ell\right)}{E_{n}} \sum_{j=0}^{\infty} \tilde{A}_{j} R_{j n}-\frac{1}{E_{n} \sinh \left(2 s_{n} \ell\right)} \sum_{j=0}^{\infty} D_{j} R_{j n} \\
B_{n} e^{2 s_{n} \ell}-C_{n} e^{-2 s_{n} \ell} & =-\frac{\operatorname{coth}\left(2 s_{n} \ell\right)}{E_{n}} \sum_{j=0}^{\infty} D_{j} R_{j n}+\frac{1}{E_{n} \sinh \left(2 s_{n} \ell\right)} \sum_{j=0}^{\infty} \tilde{A}_{j} R_{j n}
\end{aligned}
$$

where $\tilde{A}_{j}=\left(A_{j}+F_{j}\right)$. Due to the similarity in the structures of (26) and (27) and also (A.1) and (A.2), it is only necessary to derive the root-free expression for (41). The appropriate result for the power absorbed across the vertical face at $x=2 \ell$ can then be obtained by interchanging the coefficients $D_{j}$ and $\tilde{A}_{j}$ (and likewise $D_{q}$ and $\tilde{A}_{q}$ ) throughout. On eliminating $B_{n}$ and $C_{n}$ from (41) and interchanging the orders of summation, it is found 
that

$$
P_{x=0}=\Re\left\{-\frac{i}{b} \sum_{j=0}^{\infty} \sum_{q=0}^{\infty}\left(\tilde{A}_{j} \tilde{A}_{q}^{*} \sum_{n=0}^{\infty} \frac{s_{n}^{*} R_{q n}^{*} T 1_{j n}}{\tanh \left(2 s_{n}^{*} \ell\right) E_{n}^{*}}-\tilde{A}_{j} D_{q}^{*} \sum_{n=0}^{\infty} \frac{s_{n}^{*} R_{q n}^{*} T 1_{j n}}{\sinh \left(2 s_{n}^{*} \ell\right) E_{n}^{*}}\right)\right\}
$$

where

$$
T 1_{j n}=\sum_{m=0}^{\infty} \frac{\Delta_{m n} R_{j m}}{\left[\lambda_{m}^{2}-\left(\lambda_{n}^{*}\right)^{2}\right] E_{m}}=\frac{\gamma_{n}^{*} \sinh \left(\gamma_{n}^{*} a\right) Q_{j}\left(s_{n}^{*}\right)}{\left[(j \pi / b)^{2}+\left(\lambda_{n}^{*}\right)^{2}\right]}
$$

with $\Delta_{m n}$ defined by (42). Note that the right hand side of (A.4) was obtained using a similar contour integral approach to that described in section III B. It follows that

$$
P_{x=0}=\Re\left\{-\frac{i}{b} \sum_{j=0}^{\infty} \sum_{q=0}^{\infty}\left[\tilde{A}_{j} \tilde{A}_{q}^{*} S 1_{j q}^{*}-\tilde{A}_{j} D_{q}^{*} S 2_{j q}^{*}\right]\right\}
$$

where

$$
S 1_{j q}=\sum_{n=0}^{\infty} \frac{s_{n} \gamma_{n} \sinh \left(\gamma_{n} a\right) R_{q n} Q_{j}\left(s_{n}\right)}{\tanh \left(2 s_{n} \ell\right) E_{n}\left[(j \pi / b)^{2}+\left(\lambda_{n}\right)^{2}\right]}
$$

and $S 2_{j q}$ is identical in structure to $S 1_{j q}$ but with the term $\tanh \left(2 s_{n} \ell\right)$ in the denominator of the summand replaced by $\sinh \left(2 s_{n} \ell\right)$. Again, the method described in section III B can be used to express the quantities $S 1_{j q}$ and $S 2_{j q}$ in the forms (46) and (47). For $S 1_{j q}$, the appropriate integral is

$$
J_{j q}^{(S 1)}=\lim _{X \rightarrow \infty} \int_{-i X}^{i X} \frac{\ell \Upsilon(s) L_{q}(s) Q_{j}(s)}{\tanh (2 s \ell)\left[(j \pi / b)^{2}+\lambda^{2}\right]} d s .
$$

and for $S 2_{j q}$ the integral is the same except the quantity $\tanh (2 s \ell)$ in the denominator of the integrand is replaced by $\sinh (2 s \ell)$. To put (A.5) in exactly the same form as (44), it is necessary only to replace the summand with its conjugate and multiply the whole expression by -1 . Finally, as mentioned above, the equivalent expression for the power absorbed by the vertical face at $x=2 \ell$, that is $P_{x=2 \ell}$, is obtained simply by interchanging the quantities $\tilde{A}_{j}$ and $D_{j}$ (likewise $\tilde{A}_{q}$ and $D_{q}$ ).

Now consider the power absorbed by the horizontal face of the silencer lining,this is given, in terms of the roots $s_{n}, n=0,1,2, \ldots$ of the dispersion relation, by (48). On interchanging the counters in the second and fourth sums, (48) may be expressed as

$$
\begin{aligned}
P_{y=a} & =-\Re\left\{\frac{i}{b} \sum_{m=0}^{\infty} \sum_{n=0}^{\infty} \frac{\cosh \left(\gamma_{m} a\right) \gamma_{n}^{*} \sinh \left(\gamma_{n}^{*} a\right)}{s_{m}^{2}-\left(s_{n}^{*}\right)^{2}}\left[G_{m n}(\ell)-G_{m n}(0)\right]\right. \\
& \left.-\frac{i}{b} \sum_{m=0}^{\infty} \sum_{n=0}^{\infty} \frac{\cosh \left(\gamma_{n} a\right) \gamma_{m}^{*} \sinh \left(\gamma_{m}^{*} a\right)}{s_{n}^{2}-\left(s_{m}^{*}\right)^{2}}\left[G_{m n}^{*}(\ell)-G_{m n}^{*}(0)\right]\right\}
\end{aligned}
$$

where $G_{m n}(x)$ is defined in (43). 
It is intended to deal only with the first and third terms of (A.8), i.e. those containing $\ell$. The equivalent results for the second and third terms can be deduced by interchanging $\tilde{A}_{j}$ and $D_{j}$ (likewise $\tilde{A}_{q}$ and $D_{q}$ ) and changing the sign of the expression. Thus, on using (27) and (A.2) to re-express the first and third terms of (A.8) in terms of the coefficients $D_{j}$ and $\tilde{A}_{j}$, and noting that

$$
\left(\sum_{n=0}^{\infty} \frac{\gamma_{n} \sinh \left(\gamma_{n} a\right) R_{j n}}{E_{n}\left(s_{n}^{2}-\left(s_{m}^{*}\right)^{2}\right)}\right)^{*}=-\frac{\gamma_{m} \sinh \left(\gamma_{m} a\right) L_{j}^{*}\left(s_{m}\right)}{K^{*}\left(s_{m}\right)}
$$

and

$$
\sum_{n=0}^{\infty} \frac{\cosh \left(\gamma_{n} a\right) R_{j n}}{E_{n}\left(s_{n}^{2}-\left(s_{m}^{*}\right)^{2}\right)}=-\frac{\cosh \left(\gamma_{m}^{*} a\right) L_{j}\left(s_{m}^{*}\right)}{K\left(s_{m}^{*}\right)}+\frac{P_{j}\left(s_{m}^{*}\right)}{(j \pi / b)^{2}+\left(\gamma_{m}^{*}\right)^{2}}
$$

where $K\left(s_{m}^{*}\right) \neq K^{*}\left(s_{m}\right)$ and $L\left(s_{m}^{*}\right) \neq L^{*}\left(s_{m}\right)$, it is found that

$$
\begin{gathered}
\Re\left\{\frac{i}{b} \sum_{m=0}^{\infty} \sum_{n=0}^{\infty} \frac{\cosh \left(\gamma_{m} a\right) \gamma_{n}^{*} \sinh \left(\gamma_{n}^{*} a\right) G_{m n}(\ell)}{s_{m}^{2}-\left(s_{n}^{*}\right)^{2}}-\frac{\cosh \left(\gamma_{n} a\right) \gamma_{m}^{*} \sinh \left(\gamma_{m}^{*} a\right) G_{m n}^{*}(\ell)}{s_{n}^{2}-\left(s_{m}^{*}\right)^{2}}\right\} \\
=\Re\left\{\frac{i}{b} \sum_{j=0}^{\infty} \sum_{q=0}^{\infty}\left[D_{j} \tilde{A}_{q}^{*} S 3_{j q}^{*}-D_{j} D_{q}^{*} S 4_{j q}^{*}\right]\right\} .
\end{gathered}
$$

Note that, the results given in (A.9) and (A.10) are again proven using contour integration. Now, on adding in the contributions from the terms in (A.8) that do not include $\ell$, it is found that

$$
P_{y=a}=-\Re\left\{\frac{i}{b} \sum_{j=0}^{\infty} \sum_{q=0}^{\infty}\left[D_{j} \tilde{A}_{q}^{*}+D_{q}^{*} \tilde{A}_{j}\right] S 3_{j q}^{*}-\left[D_{j} D_{q}^{*}+\tilde{A}_{j} \tilde{A}_{q}^{*}\right] S 4_{j q}^{*}\right\} .
$$

On taking the complex conjugate of the summand and changing the sign of the whole expression, this is easily recognised as (49).

Note that, in (A.11), $S 4_{j q}$ is given by

$$
S 4_{j q}=\sum_{m=0}^{\infty} \frac{s_{m} \gamma_{m} \sinh \left(\gamma_{m} a\right) R_{q m} P_{j}\left(s_{m}\right)}{\tanh \left(2 s_{m} \ell\right) E_{m}\left[(j \pi / b)^{2}+\left(\gamma_{m}\right)^{2}\right]} .
$$

and $S 3_{j q}$ is identical in structure to $S 4_{j q}$ but with $\tanh \left(2 s_{n} \ell\right)$ replaced by $\sinh \left(2 s_{n} \ell\right)$. These sums can be cast in the forms given in (50) and (51) using the the integral approach described in section III B. The appropriate integrals have a similar form to (A.7).

\section{References}

[1] H. Besserer and P.G. Malischewsky, "Mode series expansions at vertical boundaries in elastic waveguides" Wave Motion, 39, 41-59 (2004). 
[2] A. Cummings and N, Sormaz, "Acoustic attenuation in dissipative splitter silencers containing mean fluid flow" J. Sound Vib. 168, 209-227 (1993).

[3] D.V. Evans and R. Porter "Wave scattering by narrow cracks in ice sheets floating on water of finite depth." Journal of fluid Mechanics, 484, 143-165 (2003).

[4] L. Huang "A theoretical study of duct noise control by flexible panels" J. Acoust. Soc. Am. 106, 1801-1809 (1999).

[5] L. Huang "Modal anaylis of a drumlike silencer" J. Acoust. Soc. Am. 112, 2014-2025 (2002).

[6] R. Kirby and A. Cummings "Prediction of the bulk acoustic properties of fibrous materials at low frequencies" Applied Acoustics, 56, 101-125 (1999).

[7] R. Kirby and J.B. Lawrie, "A point collocation approach to modelling large dissipative silencers" J sound Vib. 286, 313-339 (2005).

[8] R. Glav "The point-matching method on dissipative silencers of arbitrary crosssection" J. Sound Vib. 189, 123-135 (1996).

[9] G.A. Kriegsmann, "The flanged waveguide antenna: discrete reciprocity and conservation" Wave Motion 29, 81-95 (1999).

[10] J.B. Lawrie and I.D. Abrahams, "An orthogonality condition for a class of problems with high order boundary conditions; applications in sound/structure interaction" Q. Jl. Mech. Appl. Math., 52(2), 161-181 (1999).

[11] J.B. Lawrie and I.D. Abrahams, "On the propagation and scattering of fluidstructural waves in a three-dimensional duct bounded by thin elastic walls" Proceedings of IUTAM 2000/10, Kluwer. Eds. I.D. Abrahams, P.A. Martin, M.J. Simon. ISBN 1-4020-0590-3 (2002).

[12] F.P. Mechel, "Theory of baffle-type silencers" Acustica, 70, 93-111 (1990).

[13] A. Selamet, M.B. Xu, I.-J. Lee and N.T. Huff, "Analytic approach for sound attenuation in perforated dissipative silencers" J. Aoust. Soc. Am 115(5), 2091-2099 (2004). 
[14] D.P. Warren, J.B. Lawrie and I.M. Mohamed, "Acoustic scattering in wave-guides with discontinuities in height and material property" Wave Motion, 36(2), 119-142 (2002). 
Table I: Silencer one, EMED forcing: comparison of transmission loss data for the "rootfree", "rooty" and point-collocation methods.

\begin{tabular}{|l|l|l|l|}
\hline \multicolumn{4}{|c|}{ Silencer 1: EMED forcing } \\
\hline Frequency & Non-rooty & Rooty & Point Collocation \\
\hline 63 & 16.34688936 & 16.34478568 & 16.56900793 \\
125 & 27.24962896 & 27.24679555 & 27.30626097 \\
250 & 46.21350006 & 46.21352061 & 46.18514037 \\
500 & 53.41436969 & 53.41410153 & 53.40450771 \\
1000 & 50.01804544 & 50.01620733 & 50.00904946 \\
2000 & 19.11579843 & 19.11573366 & 19.11350138 \\
4000 & 8.947613426 & 8.948377897 & 8.946284148 \\
\hline
\end{tabular}

Table II: Silencer two, EMED forcing: comparison of transmission loss data for the "root-free", "rooty" and point-collocation methods.

\begin{tabular}{|l|l|l|l|}
\hline \multicolumn{4}{|c|}{ Silencer 2: EMED forcing } \\
\hline Frequency & Non-rooty & Rooty & Point Collocation \\
\hline 63 & 1.828077049 & 1.829027604 & 1.85508498 \\
125 & 6.784509519 & 6.78685205 & 6.822066684 \\
250 & 20.63940465 & 20.64184964 & 20.69345116 \\
500 & 11.40109296 & 11.40130263 & 11.4015143 \\
1000 & 5.355438508 & 5.355675135 & 5.356555514 \\
2000 & 5.125842846 & 5.125912909 & 5.126296988 \\
4000 & 5.504838985 & 5.504690444 & 5.504658887 \\
\hline
\end{tabular}




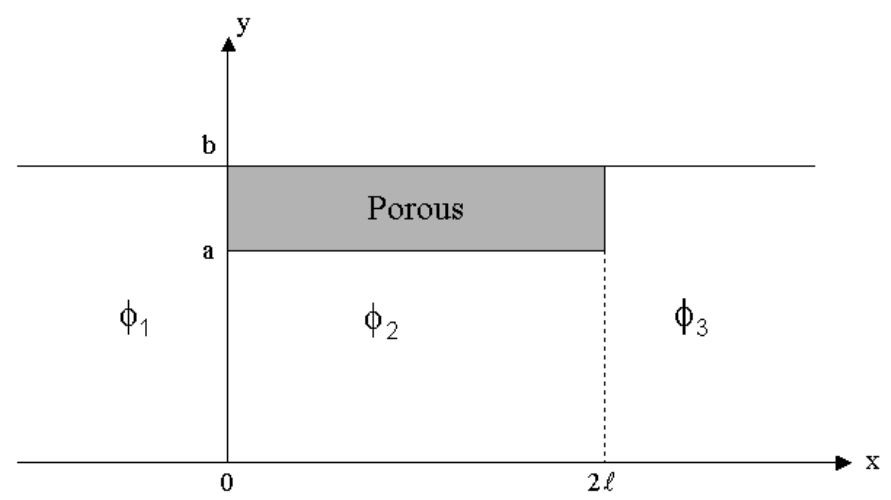

Figure 1: Silencer geometry.

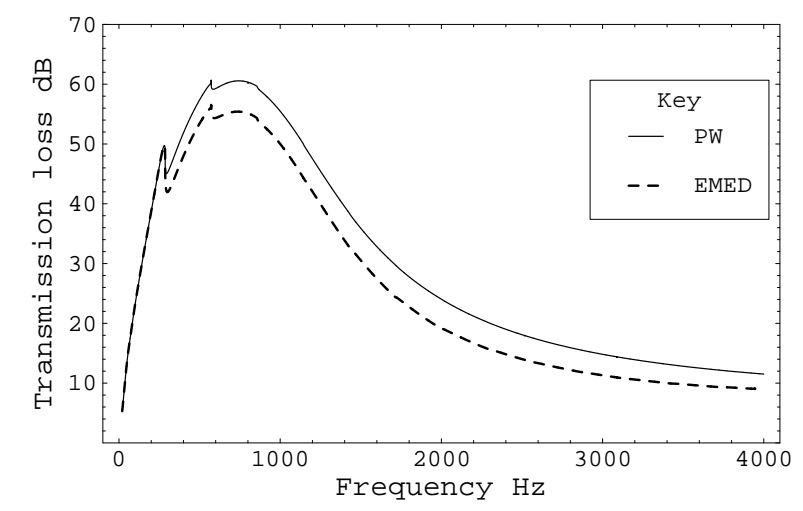

Figure 2: Transmission loss against frequency for silencer one. Both plane wave and EMED forcing are depicted.

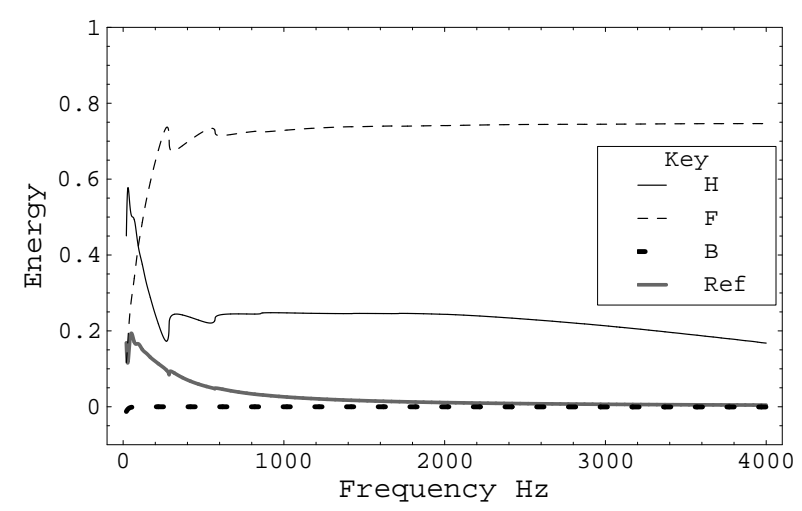

Figure 3: Proportion of energy reflected and absorbed across each surface of silencer one for plane wave forcing. The $x=0, y=a$ and $x=2 \ell$ surfaces of the silencer are denoted by $\mathrm{F}, \mathrm{H}$ and $\mathrm{B}$ respectively. Ref indicates the reflected component of power. 


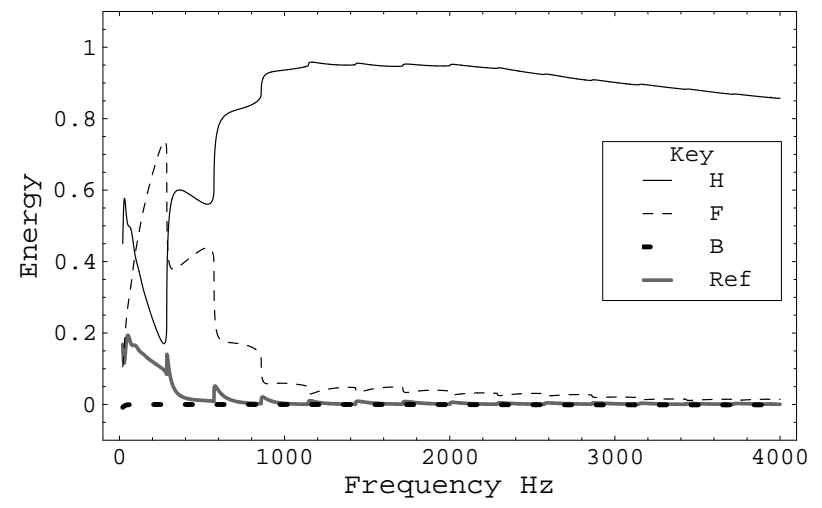

Figure 4: Proportion of energy reflected and absorbed across each surface of silencer one for EMED forcing. The $x=0, y=a$ and $x=2 \ell$ surfaces of the silencer are denoted by F, H and B respectively. Ref indicates the reflected component of power.

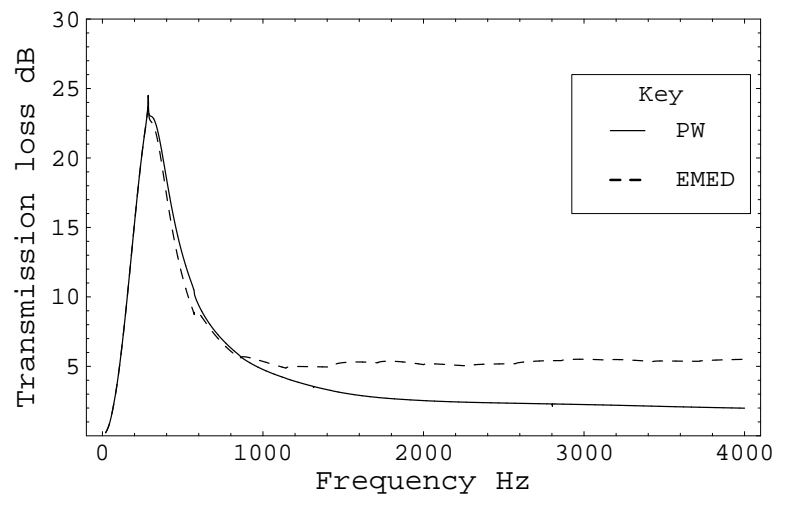

Figure 5: Transmission loss against frequency for silencer two. Both plane wave and EMED forcing are depicted. 


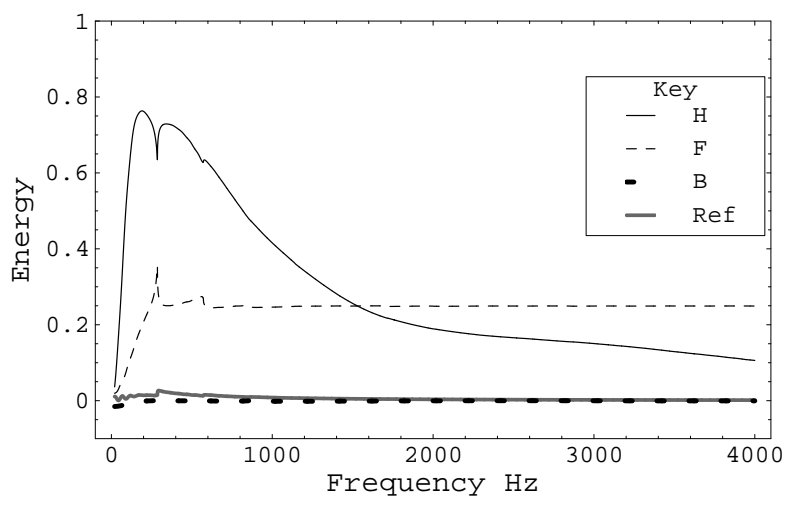

Figure 6: Proportion of energy reflected and absorbed across each surface of silencer two for plane wave forcing. The $x=0, y=a$ and $x=2 \ell$ surfaces of the silencer are denoted by $\mathrm{F}, \mathrm{H}$ and $\mathrm{B}$ respectively. Ref indicates the reflected component of power.

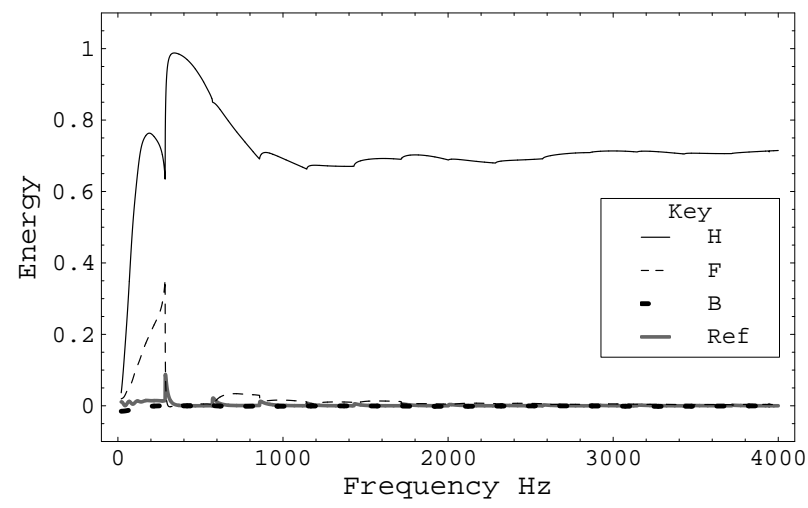

Figure 7: Proportion of energy reflected and absorbed across each surface of silencer two for EMED forcing. The $x=0, y=a$ and $x=2 \ell$ surfaces of the silencer are denoted by $\mathrm{F}, \mathrm{H}$ and $\mathrm{B}$ respectively. Ref indicates the reflected component of power. 